\title{
On the Orbital Stability of Pendulum-Like Vibration of a Rigid Body Carrying a Rotor
}

\author{
H.M. Yehia ${ }^{\mathrm{a}}$, E.G. El-Hadidy ${ }^{\mathrm{b}}$. \\ ${ }^{a}$ Mathematics Department, Faculty of Science, Mansoura University, Mansoura 35516, Egypt. \\ ${ }^{b}$ Mathematics Department, Faculty of Science, Damietta University, New Damietta, Egypt.
}

*Corresponding author (email: eman_elhadidy@yahoo.com)

\begin{abstract}
One of the most notable effects in mechanics is the stabilization of the unstable upper equilibrium position of a symmetric body fixed from one point on its axis of symmetry, either by giving the body a suitable angular velocity or by adding a suitably spinned rotor along its axis. This effect is widely used in technology and in space dynamics. The aim of the present article is to explore the effect of the presence of a rotor on a simple periodic motion of the rigid body; its motion as a physical pendulum. The equation in the variation for pendulum vibrations takes the form

$\frac{d^{2} \gamma_{3}}{d u^{2}}+\alpha\left(\alpha v^{2}+\frac{1}{2}+\rho^{2}-(\alpha+1) v^{2} s n^{2} u+2 v \rho \sqrt{\alpha} c n u\right) \gamma_{3}=0$

in which $\alpha$ depends on the moments of inertia, $\rho$ on the gyrostatic momentum of the rotor and $v$ (the modulus of the elliptic function) depends on the total energy of the motion. This equation, which reduces to Lame's equation when $\rho=0$ was not studied to any extent in the literature. The determination of the zones of stability and instability of plane motion reduces to finding conditions on those parameters for existence of primitive periodic solutions (with periods $4 \mathrm{~K}(\mathrm{v}), 8 \mathrm{~K}(\mathrm{v}))$. Full analysis of primitive periodic solutions of this equation is performed analogous to that of Ince for Lame's equation. Zones of stability and instability are determined analytically and illustrated in a graphics form by plotting surfaces separating them in the three dimensional space of parameters. The problem is also solved numerically on certain sections of the parameter space and results are compared to analytical ones.
\end{abstract}

Keywords: pendulum-like motion, plane motion, orbital stability, gyrostat, rigid body with a rotor

\section{Introduction}

It was proved by Kovalevskaya that the solution of Euler-Poisson equations of motion of the rigid body about a fixed point can be expressed as meromorphic functions in time only in the three famous general integrable cases of Euler, Lagrange and the case which became known as Kovalevskaya's case [1] (see also [2]). Only in these same cases we have a fourth first integral of the Euler-Poisson's equations of motion as an algebraic (in fact, polynomial) function in EulerPoisson's variables. A set of particular solutions, corresponding to some restrictions imposed on the initial conditions of the motion in addition to those on the distribution of mass in the body was obtained by several authors, e.g. Goryachev and Chaplygin, Hess, Grioli, Bobylev and Steklov [2].

It is of great importance, with regard to the 
practical applications of the problem, to perform a detailed qualitative analysis of some simple motions, such as steady motions or pendulum motions. Such analysis is done only for a few cases. Noting that only stable motion and equilibria can be realized due to the inevitable deviations in the initial conditions and in the determination of the distribution of mass in the body, the study of stability acquires an increasing importance. The study of Staude (permanent) rotations of the heavy rigid body about a fixed point was partially studied by Grammel [3] and in more detail by Rumyantsev [4]. The effect of damping was considered by Schiehlen [5]. The simplest case, after the permanent rotation, is the plane motion about a fixed horizontal axis, like a compound pendulum. These motions are either oscillations or rotations, according to the total energy of the body and they are periodic in time. The equations in the variations of periodic motions are linear equations with periodic coefficients. Thus, for the study of stability of a periodic motion of the rigid body we deal with six equations with periodic coefficients, which admit three integrals. The study of such system is quite difficult and can be performed mainly numerically.

It is evident that the Euler-Poisson system of equations of motion of the rigid body can be reduced to a single second-order differential equation, by using the three known general integrals. However, several trials by various authors have failed to realize this maximal reduction because of great algebraic difficulties encountered [6], [7], [8], [9] or led to equations that are so complicated that they were never used in any application [10]. In 1976, Yehia solved the problem of maximal reduction of the equations of motion to a single second order differential equation involving isometric variables on the ellipsoid of inertia [11]. In 1983, he achieved reduction to a second-order equation involving two geometrical variables, namely, two components of the vector $\boldsymbol{\gamma}$ constant in space, referred to principal axes of inertia of the body [12].

Being symmetric, transparent and relatively compact, this reduced equation turned out to be very useful in the study of stability of periodic motions. It was used as a basis of the stability analysis of plane (pendulum-like) motions of the rigid body in uniform gravity [13], [14] and in the approximate Newtonian gravitational field [15]. Plane motions and their stability were considered in some later papers on the basis of
Euler-Poisson equations. Thai analyzed numerically the case of vibration for three values of energy parameter [16]. Dovbysh investigated some qualitative properties of rotational motions [17]. Markeev studied the stability of pendulum motions not only in the linear approximation [18] but also in the nonlinear setting, using reduction of the Hamiltonian to the Birkhoff normal form. However, this was done mainly for bodies, whose distribution of mass satisfies conditions of intgerable cases: for Kovalevskaya's case [19] and for the case of Goryachev and Chaplygin [20]. Stability analysis was performed also for some other known periodic solutions of the Euler-Poisson equations of motion. Grioli's regular processional motion is one of the most famous periodic solutions. Its stability was considered in Grioli's original 1947 paper [21] and later by other authors [22], [23] in a restricted setting. Tkhai studied the full problem numerically and obtained partial results [24]. The most exhaustive analysis of the stability of Grioli's motion was performed by Markeev in two papers [25], [26]. Zones of stability and instability were presented in the plane of the two parameters that determine the state of the system. Markeev also studied the stability of the periodic solution of Euler-Poisson equations known after Steklov [27]. The problem involves also two parameters and results are presented in their plane. In this paper we consider the stability of plane vibrational motion for dynamically symmetric body in presence of gyrostatic moment. The picture of zones of stability and instability is explored in the 3D space of parameters of the problem.

\section{The description of pendulum-like motions}

Consider the mechanical system known as the gyrostat composed of a rigid body $S_{0}$, fixed from a single point $O$, and a symmetric rotor $S_{1}$ fixed from its axis of symmetry in the carrier body $S_{0}$ by means of a cylindrical hinge and rotating with a constant angular speed relative to $S_{0}$. Let $O X Y Z$ be an inertial reference frame with $Z$-axis directed vertically upwards and Oxyz be the system of axes fixed in the body and coinciding with its principal axes at the fixed point. We introduce the following notation, where all vectors are referred to the system of principal axes $O x y z: \omega=(p, q, r)$ is the angular velocity of the carrier body $S_{0}, \gamma=\left(\gamma_{1}, \gamma_{2}, \gamma_{3}\right)$ 
is the unit vector in the upward vertical direction, $\mathbf{k}=\left(k_{1}, k_{2}, k_{3}\right)$ is the angular momentum of the rotor relative to $S_{0}$ (called also by many authors the gyrostatic moment), constant in $S_{0}, \mathbf{r}_{0}$ $=\left(x_{0}, y_{0}, z_{0}\right)$ is the position vector of the centre of mass of the whole system, $A, B$ and $C$ are the principal moments of inertia of the system.

The equation of motion of the carrier-body $S_{0}$ is usually written in the Euler-Poisson form [2]:

$$
\begin{aligned}
& A \dot{p}+(C-B) q r+q k_{3}-r k_{2}=M g\left(z_{0} \gamma_{2}-y_{0} \gamma_{3}\right), \\
& B \dot{q}+(C-A) p r+r k_{1}-p k_{3}=M g\left(x_{0} \gamma_{3}-z_{0} \gamma_{1}\right), \\
& C \dot{r}+(B-A) q p+p k_{2}-q k_{1}=M g\left(y_{0} \gamma_{1}-x_{0} \gamma_{2}\right), \\
& \dot{\gamma}_{2}+r \gamma_{1}-p \gamma_{3}=0, \\
& \quad \dot{\gamma}_{3}+p \gamma_{2}-q \gamma_{1}=0 . \\
& \quad \dot{\gamma}_{1}+q \gamma_{3}-r \gamma_{2}=0,
\end{aligned}
$$

where dots denote differentiation with respect to time. This system of equations admits the well-known three classical integrals [2]:

Energy integral:

$\frac{1}{2}\left(A p^{2}+B q^{2}+C r^{2}\right)+M g\left(x_{0} \gamma_{1}+y_{0} \gamma_{2}+z_{0} \gamma_{3}=h\right.$.

Area integral:

$\left(A p+k_{1}\right) \gamma_{1}+\left(B q+k_{2}\right) \gamma_{2}+\left(C r+k_{3}\right) \gamma_{3}=f$.

Geometrical integral:

$$
\gamma_{1}^{2}+\gamma_{2}^{2}+\gamma_{3}^{2}=1 \text {, }
$$

where $h$ and $f$ are the energy and area constants, respectively.

The plane motion of a rigid body is the motion when all the elements of the body move parallel to a fixed plane it is possible only when the centre of mass of the body is in one of the principal planes of inertia. This is the type of motion that will be considered in this paper. We deal with the case of dynamically symmetric body (i.e. $\mathrm{B}=\mathrm{A}$ ) and without loss of generality, we assume that its centre of mass lies on the $x$ axis. The rotor is directed along the axis of symmetry of the body, i.e.

$$
k_{1}=k_{2}=y_{0}=z_{0}=0 \text {. }
$$

In this case Euler-Poisson's equations admits the particular solution

$$
p=q=\gamma_{3}=0,
$$

And $r, \gamma_{1}, \gamma_{2}$ are given by the equations

$$
C \dot{r}=-a \gamma_{2} \text {, }
$$

$$
\begin{aligned}
& \dot{\gamma}_{1}=r \gamma_{2}, \\
& \dot{\gamma}_{2}=-r \gamma_{1} .
\end{aligned}
$$

Substituting from (5) and (6) in (2)-(4) the three integrals of Euler-Poisson's equations take the form:

$$
\begin{aligned}
& \frac{1}{2} C r^{2}+a \gamma_{1}=h, \mathbf{a}=M g x_{0}, \\
& f=0, \\
& \gamma_{1}^{2}+\gamma_{2}^{2}=1 .
\end{aligned}
$$

From (7) one may write

$$
\gamma_{1}=-\cos \theta \quad, \gamma_{2}=\sin \theta, \quad r=\dot{\theta} \text {. }
$$

Thus the equation (8) gives

$$
\frac{t-t_{0}}{\sqrt{2 C}}=\int_{0}^{\phi=a / 2} \frac{d \phi}{\sqrt{h+a-2 a \sin ^{2}(\phi)}},
$$

\section{Variational equation in the case of vibration}

In vibration motion $-a<h<a$, consequently (11) takes the form:

$$
\sqrt{\frac{a}{C}}\left(t-t_{0}\right)=\int_{0}^{\phi} \frac{d \phi}{\sqrt{v^{2}-\sin ^{2} \phi}},
$$

where $v^{2}=\frac{h+a}{2 a}$. Substituting in (12) with $v \xi=\sin \phi \quad$ we get:

$$
\sqrt{\frac{s}{C}}\left(t-t_{0}\right)=\int_{0}^{\xi} \frac{d \xi}{\sqrt{\left(1-\xi^{2}\right)\left(1-v^{2} \xi^{2}\right)}},
$$

so that

$$
\xi=\operatorname{sn}(u, v), \quad u=\sqrt{\frac{a}{C}}\left(t-t_{0}\right)
$$

and the solution of Euler-Poisson equations describing the pendulum-like vibration can be written as:

$$
\begin{aligned}
& p=q=\gamma_{3}=0, \\
& r=2 \sqrt{\frac{h+a}{2 C}} c n(u, v), \\
& \gamma_{1}=-\left(1-2 v^{2} s^{2}(u, v)\right), \\
& \gamma_{2}=2 v \operatorname{sn}(u, v) d n(u, v) .
\end{aligned}
$$

where $s n, c n, d n$ are Jacobi's elliptic functions with modulus (e.g. [28]). Note that the solution is periodic with period $4 K(v) ; K$ being the elliptic integral of the first kind. Fig. 1 illustrates the configuration of the system described in the last paragraph, the positions of the rotor and the centre of mass relative to the inertia spheroid of the body. 


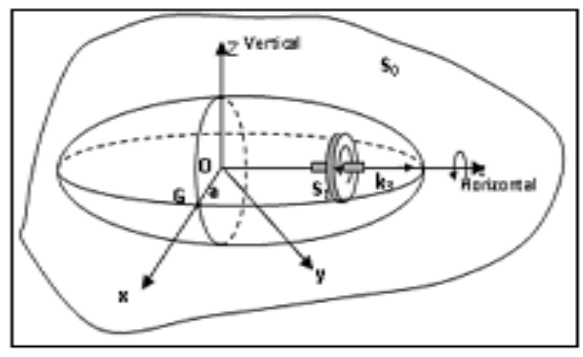

In [12], Yehia obtained the reduced equation using the known three general integrals of motion in terms of two of the components of vector $\gamma$ as variables. For the problem under consideration, in view of the symmetry of the body $B=A$ and in virtue of (5) and (9), this equation takes the form

Fig. 1 the configuration of the system

$$
\begin{aligned}
& A^{2} C \gamma_{2}^{2} D \gamma_{3}^{\prime \prime}+A^{2} C^{2}\left(1-\gamma_{3}^{2}\right) \gamma_{3}-A^{2} C \gamma_{1}\left[A-(A+2 C) \gamma_{3}^{2}\right] \gamma_{3}^{\prime}+ \\
& A^{2} C \gamma_{3}\left[C-(C+2 A) \gamma_{1}^{2}\right] \gamma_{3}^{\prime 2}-A^{3} C\left(1-\gamma_{1}^{2}\right) \gamma_{1} \gamma_{3}^{\prime 3} \\
& -\frac{\delta}{D}\left\{C \gamma_{3}\left[A(A-C)\left(1-\gamma_{3}^{2}\right)\right]+A \gamma_{1}\left[C(A-C) \gamma_{3}^{2}\right] \gamma_{3}^{\prime}\right\} \\
& +\frac{\delta}{2(U+h)}\left[\left(\mu+v \gamma_{3}^{\prime}\right) \frac{\partial U}{\partial \gamma_{1}}-\left(\lambda+\mu \gamma_{3}^{\prime}\right) \frac{\partial U}{\partial \gamma_{3}}\right]+\frac{1}{\sqrt{2(U+h)}}\left(\frac{\delta}{D}\right)^{3 / 2}\left\{2 A C k_{3} \gamma_{3}\right\}=0,
\end{aligned}
$$

where:

$$
\begin{aligned}
& D=A+(C-A) \gamma_{3}^{2}, \\
& \mu=A C \gamma_{1} \gamma_{3}, \\
& v=A\left[A\left(1-\gamma_{1}^{2}\right)+(C-A) \gamma_{3}^{2}\right], \\
& \lambda=C A\left(1-\gamma_{3}^{2}\right), \\
& \delta=\lambda+2 \mu \gamma_{3}^{\prime}+v \gamma_{3}^{\prime 2}, \\
& U=-a \gamma_{1}-\frac{k_{3}^{2} \gamma_{3}^{2}}{2\left[A+(A-C) \gamma_{3}^{2}\right]},
\end{aligned}
$$

$\gamma_{2}$ stands for $\gamma_{2}=\left(1-\gamma_{1}^{2}-\gamma_{3}^{2}\right)^{1 / 2}$, the dash denotes differentiation with respect to $\gamma_{1}$.

\section{The orbital stability problem}

The whole dynamics of the rigid body-gyrostat acted upon by potential forces is reduced to the 2nd order differential equation each solution curve

$$
\gamma_{3}=\gamma_{3}\left(\gamma_{1}\right)
$$

of this equation corresponds to a solution of the Euler-Poisson's equations. A closed curve corresponds a time periodic solution $(\omega, \gamma)$ of the Euler-Poisson's equations (with possibly-infinite period). Equation (15) admits a solution $\gamma_{3}=0$ which corresponds a motion of the body about its third principal axis while this axis preserves a fixed horizontal position. By orbital stability of pendulum motion we mean stability of the motion with respect to the variable $\gamma_{3}$. This means stable motions are those for which $\gamma_{3}$ remains small after perturbing the solution $\gamma_{3}=0$, while preserving the values of the energy and areas parameters $h$ and $f$. We note that this concept of stability is geometric and means that the axis of rotation of the pendulum after perturbation remains near to the horizontal plane in which it existed in the unperturbed motion. Substituting from (16) and linearizing in $\gamma_{3}$, equation (15) takes the form:

$$
\begin{aligned}
& \frac{d^{2} \gamma_{3}}{d \gamma^{2}}+\left[\frac{1 / 2}{\gamma-1}+\frac{1 / 2}{\gamma+1}+\frac{1}{2} \frac{1}{\left(\gamma-\frac{h}{a}\right)}\right] \frac{d \gamma_{3}}{d \gamma} \\
& +\left\{\frac{C^{2}}{2 A^{2}}\left(\frac{1}{\gamma+1}-\frac{1}{\gamma-1}\right)-\frac{C}{2 A} \frac{\gamma}{\left(\gamma^{2}-1\right)\left(\gamma-\frac{h}{a}\right)}\right. \\
& \left.+\frac{C}{2 a A^{2}} \frac{k^{2}}{\left(\gamma^{2}-1\right)\left(\gamma-\frac{h}{a}\right)}-\frac{2 k_{3} C \sqrt{C}}{A^{2}\left(\gamma^{2}-1\right) \sqrt{2 a\left(-\gamma+\frac{h}{a}\right)}}\right\}=0 .
\end{aligned}
$$

Substituting from (14) in (18), the equation in the case of vibration takes the final form:

$$
\begin{aligned}
& \frac{d^{2} \gamma_{3}}{d u^{2}}+\alpha\left(\alpha v^{2}+\frac{1}{2}+\rho^{2}-(\alpha+1) v^{2} s^{2} u\right. \\
& +2 v \rho \sqrt{\alpha} \text { cnu }) \gamma_{3}=0
\end{aligned}
$$

where $\alpha=\frac{2 C}{A}, \rho=\frac{k_{3}}{\sqrt{2 A a}}$, and $u$ is related to time by (13).

Equation, (19) involves three parameters 
$\alpha, \nu, \rho$. Due to the triangle inequalities satisfied by moments of inertia, $\alpha$ is restricted to the interval $(0,4]$. The face $\alpha=0$ is excluded since the body turns on it into a straight rod and its centre of mass cannot remain outside it. The modulus of elliptic functions $v \in[0,1)$. The third parameter can take any real value, but one can show that it suffices to consider only $\rho \geq 0$. In fact, reversing the sign of $\rho$ together with a shift $2 K$ in the argument $u$ leaves equation (19) invariant. This means that the picture of stability zones is symmetric with respect to the sign of the gyrostatic moment. Thus, the physically admissible region of the parameter space is given $R=\{\alpha, v, \rho: 0<\alpha \leq 4,0 \leq v<1, \rho \geq 0\}$.

This equation was first derived in [12], where the preliminary stability analysis was performed for very small oscillations i.e. for infinitesimal values of the modulus $v$ as for as we known, equation (19) was not investigated in the literature. Nothing is known about the analytical and qualitative properties of its solution for arbitrary values of the parameters.

\section{Primitive periodic solutions of the stability equation}

In case of absence of gyrostatic moment, equation (19) reduces to Lame's equation. In this case (19) is $4 K$ - periodic while Lame's equation (when $k_{3}=0$ ) is $2 K$ - periodic. This leads to great qualitative difference from the previous case. According to Floquet's theory [30] the study of the stability of linear differential equation with periodic coefficient reduces to finding primitive periodic solutions, since the space of parameters $\alpha, v, \rho$ is divided into distinct zones of stability and instability, separated by a certain set of boundaries corresponding to the four types of periodic solutions of the equation of variations. To find the periodic solutions of (19) is, in general, a hard task. We shall apply the procedure used by Ince for Lame's equation [31]. This will be done below the period of equation (19) is $4 K$ so its solution must be taken with period $4 K(v), 8 K(v)$. In this paper we use expansion in trigonometric series in the amplitude which are much more convenient than direct use of elliptic functions. We first transform this equation to the trigonometric form through the substitution: .

$$
\begin{aligned}
& \operatorname{snu}=\sin \phi, \quad \phi=a m u \\
& \left(1-v^{2} \sin ^{2} \varphi\right) \frac{d^{2} \gamma_{3}}{d \phi^{2}}-v^{2} \sin \phi \cos \phi \frac{d \gamma_{3}}{d \phi} \\
& +\left(H+\frac{1}{2} \alpha(\alpha+1) v^{2} \cos 2 \phi+2 v \rho \alpha^{\frac{3}{2}} \cos \phi\right) \gamma_{3}=0,
\end{aligned}
$$

where:

$$
H=\frac{1}{2} \alpha+\rho^{2} \alpha+\frac{1}{2} \alpha(\alpha-1) v^{2} .
$$

\section{Primitive periodic solutions of the stability equation (19)}

\section{$4 K$ - Periodic solution}

Such solution can be expressed as Fourier series, solution of the trigonometric form (20)

$$
\gamma_{3}=\sum_{i=0}^{\infty}\left(A_{i} \cos (i \phi)+B_{i} \sin (i \phi)\right)
$$

Substituting from (21) in(20), equating the coefficients of sine and cosine functions to zero, we have an infinite system of equations for $A_{i}$ and $B_{i}$. In this way we obtain a system that can be split into two systems i.e.,

$$
\begin{aligned}
& A(\alpha, v, \rho) \mathbf{v}=0, B(\alpha, v, \rho) \mathbf{w}=0, \\
& \text { where } A(\alpha, v, \rho), B(\alpha, v, \rho) \text { are square }
\end{aligned}
$$
penta-diagonal matrices of infinite dimension and $\mathbf{v}$ is an infinite column vector where the elements are coefficients of cosine (20) and w is an infinite column vector where the elements are coefficients of sine (20). To have a non-trivial solution, the determinants of $A$ and $B$ must be equal zero, giving a condition in the form

$$
f(\alpha, v, \rho)=0 \text {, }
$$

which determines some surfaces in the $\alpha v \rho$ space on which odd and even periodic solutions of period $4 K$ for (19) exist. Of course, it is difficult to calculate a determinant of the infinite matrix. So we should truncate the infinite sequence of equations after the $m$-th equations, where $m$ is a suitably large number. From a technical point of view, it follows that, if more modes are added the result will be more accurate. The calculations can be reasonably done with the modern computer algebra systems such as Maple. We consider the determinant of a pentadiagonal matrix of the form:

In the case of $4 K$ periodic solution:

1- The determinantal equation for the even solutions 


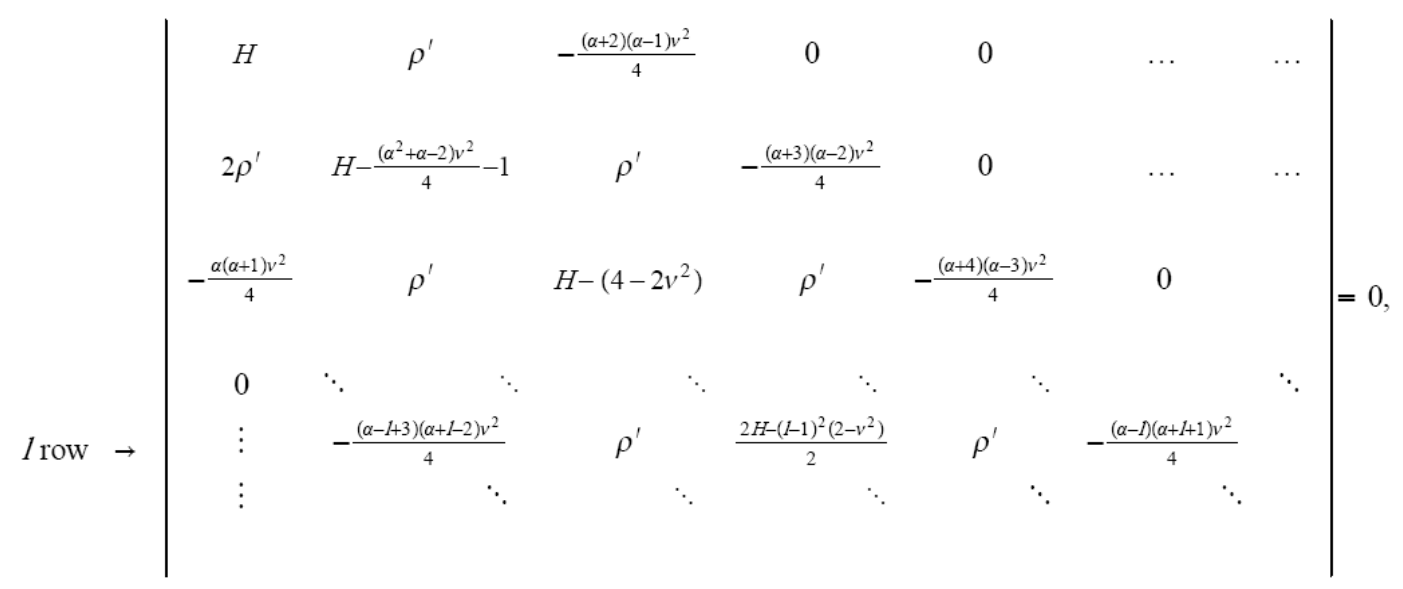

where $\rho^{\prime}=\rho v \alpha^{\frac{3}{2}}, \quad l=3,4,5, \ldots$, we assume that $a_{l j}=0$ if $|l-j|>2$, where $a_{l j}$ is the element in the row $l$ and column $j$.

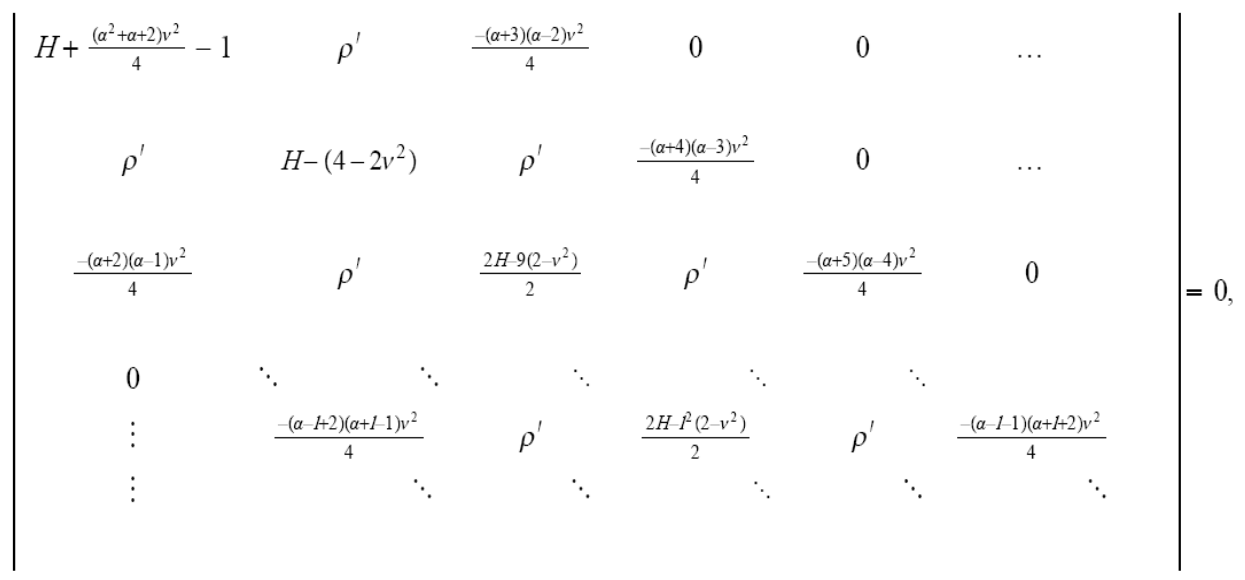

\section{$8 K$ - Periodic solution}

Such solution can be expressed as a $4 \pi$ periodic Fourier series, solution of the trigonometric form (20)

1-The determinantal equation for the even solutions:

$$
\gamma_{3}=\sum_{i=0}^{\infty}\left(A_{i} \cos \left(i-\frac{1}{2}\right) \phi+B_{i} \sin \left(i-\frac{1}{2}\right) \phi\right)
$$

Substituting from (25) in (20), yields in the same manner as before.

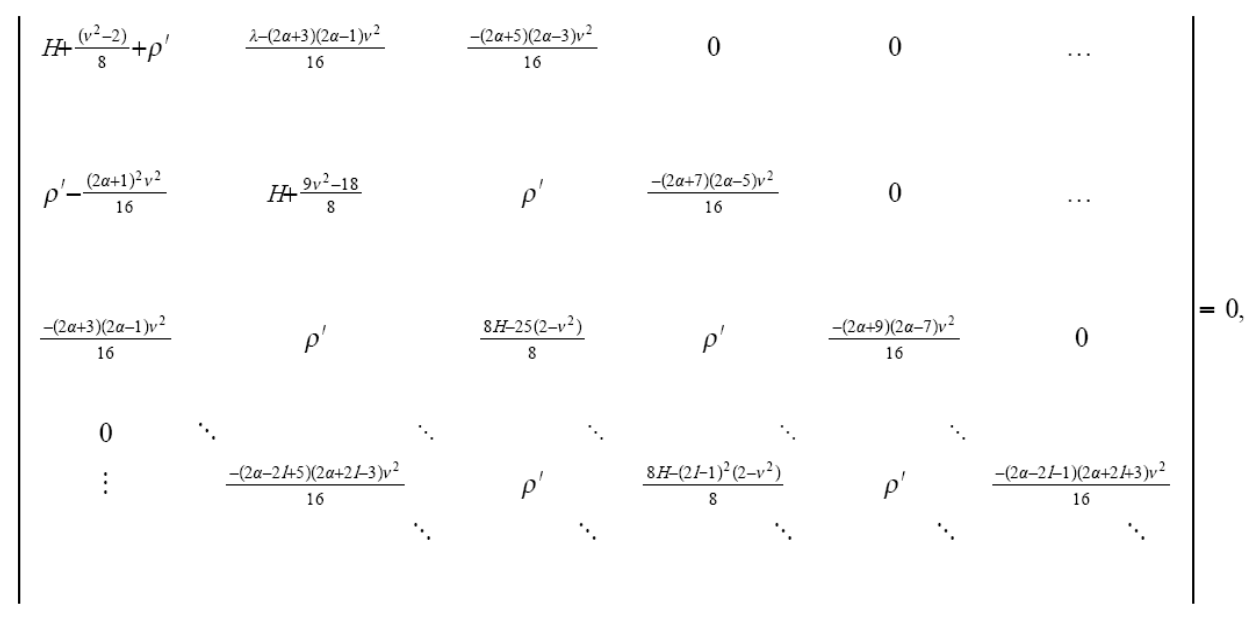


2- The determinantal equation for the odd solutions:

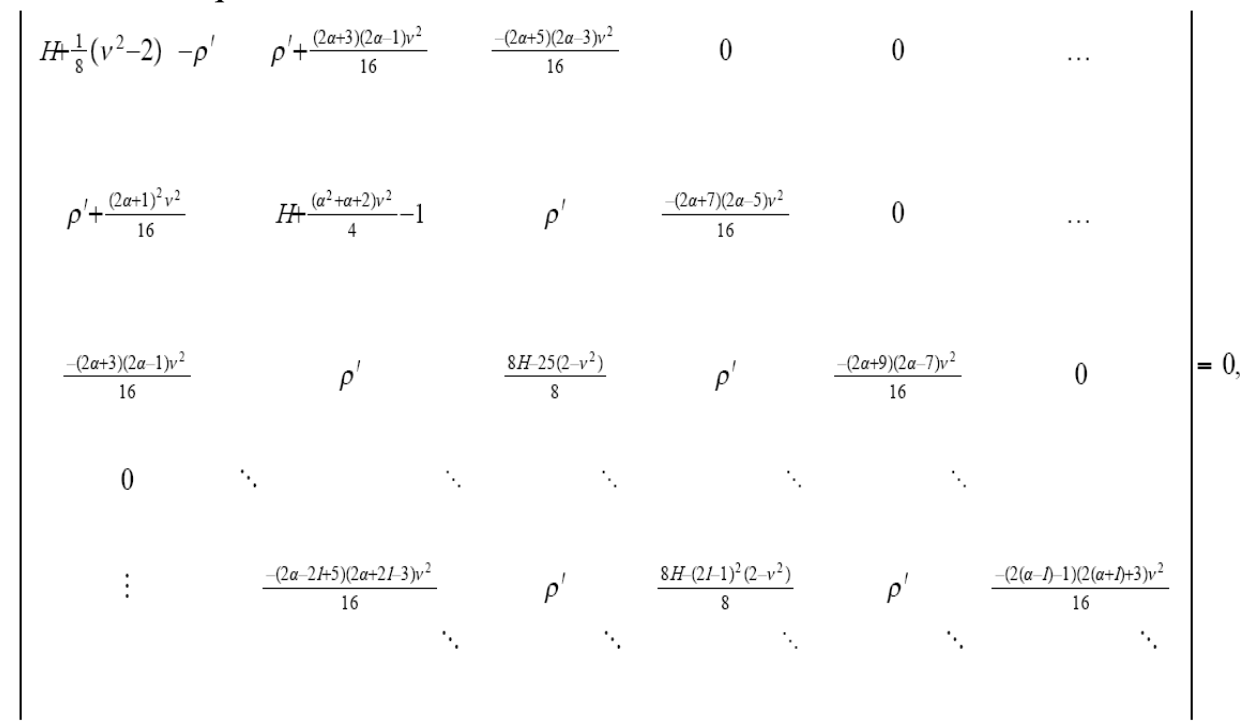

\section{The stability picture}

$v=0$ of the region $R$. It takes the form

$$
\frac{d^{2} \gamma_{3}}{d u^{2}}+\alpha\left(\frac{1}{2}+\rho^{2}\right) \gamma_{3}=0 \text {. }
$$

Fig. 2 shows the boundary surfaces of the four parameters $(\alpha, \rho, v)$ from two different eye positions.

It is readily seen that the growth of the gyrostatic moment of the rotor (proportional to the parameter $\rho$ ) has the effect of pressing the zones of stability and instability towards the left face $\alpha=0$ of the admissible region $R$, while new zones enter this region through the right face $\alpha=4$. This conclusion may be augmented by

As shown in Fig. 3, this face is divided by the curves $\alpha\left(\frac{1}{2}+\rho^{2}\right)=\left(\frac{n}{2}\right)^{2} \quad(n \quad$ integer $)$ into separate zones of stability, i.e. we have a countable set of different stability zones, no instability zone is present. For small values of $v>0$, an instability zone appears near to each of those curves, which splits on the neighbouring sections to two curves carrying odd or even solutions.
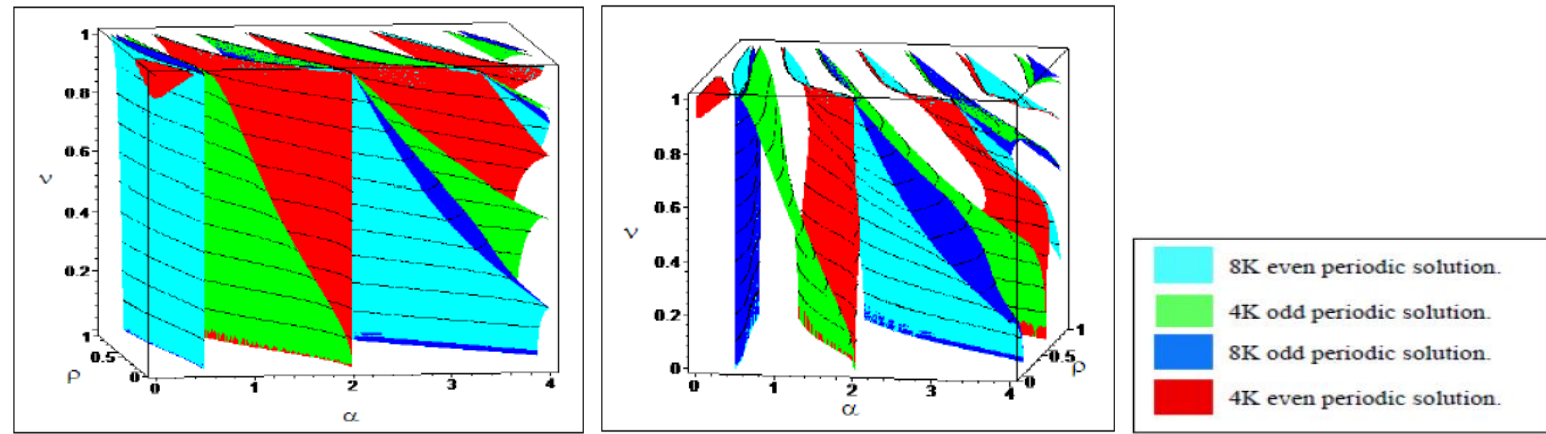

Fig. 2 a: full picture. b: full picture from a different eye position

As seen from Fig. 2 and 3, the stability zones begin wide and cover all the bottom face and end at the top as narrow wedges. The instability zones, conversely, begin as narrow wedges at the bottom and grow to include all the top area. From Fig. 3 and 4 we note that a single instability zone disappears as $\rho$ increases. That is the small zone at the upper left front corner of $R$. To follow this up we consider the equation (19) near the face $\alpha=0$. On that face, the only periodic solution of (19) is the constant solution. This indicates that this line may form, or it is a part of, a boundary between two of the stability and instability zones. One solution of (19) must be $4 \mathrm{~K}$-periodic on this boundary. We try an expansion for this solution in the neighbourhood of the section $\alpha=0$ : 


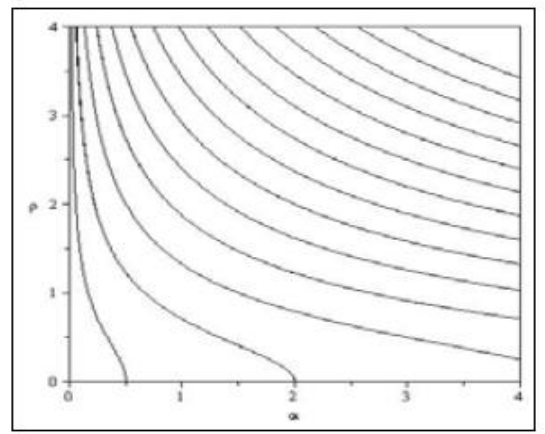

Fig. 3 The bottom face $v=0$

$$
\gamma_{3}=y_{0}+\alpha y_{1}+\alpha^{2} y_{2}+\ldots
$$

Substituting in (19) we get for the first two powers of $\alpha$ :

$$
y_{0}^{\prime \prime}=0, y_{1}^{\prime \prime}+y_{0}\left(\frac{1}{2}+\rho^{2}-v^{2} s n^{2} u\right)=0 .
$$

The periodic solution of the first equation is $y_{0}=$ const. and the condition for $4 K$ periodicity of the solution of the second one is:

$$
\int_{0}^{4 K}\left(\frac{1}{2}+\rho^{2}-v^{2} s n^{2} u\right) d u=0,
$$

or equivalently,

$$
\left(-\frac{1}{2}+\rho^{2}\right) K(v)+E(v)=0 .
$$

Where $K(v)$ is the complete elliptic integral of the first kind with modulus $v, E(v)$ is the complete elliptic integral of the second kind with modulus $v$. The last equation has a real solution $v(\rho)$ on the interval $\left[0, \frac{1}{\sqrt{2}}=0.7071\right]$ with $\quad v(0)=0.9089$ and $\quad v\left(\frac{1}{\sqrt{2}}\right)=1$. This solution is shown in Fig. 4. The above mentioned little (pocket) of instability completely disappears by $\rho$ reaching $\frac{1}{\sqrt{2}}$.

On the front face $\rho=0$, equation (19) takes the form of Lame equation and the stability zones were found in [12]. They have the shape shown in Fig. 5-a. Three zones of stability and four zones of instability of Lame's equation are present, separated by curves carrying $2 K$ and $4 K$-periodic even and odd solutions. For $\rho>0$, equation (19) is $4 K$-periodic and the four types of solutions are deformed into even and odd $4 K$ -periodic solutions. The second primitive periodic solutions of (19) are odd and even $8 K$ periodic solutions. In general those solutions exist on pairs of different surfaces, which meet the face $\rho=0$, each pair at a single line lying in the middle of one of the stability zones and known to carry simultaneously even and odd $8 \mathrm{~K}$ -periodic solutions of Lame equation [31] ${ }^{1}$. The last lines are represented by solid lines in Fig. 5a. For special values of $\rho$ $(\rho=0.1,0.2,0.3,0.5,0.7,1) \quad$ the zones of stability are shown in Fig. 5-b,c, d,e,f,g.

${ }^{1}$ For $\rho=0, \alpha=\frac{1}{2}$ equation (19) reduces to Lame equation $\frac{d^{2} \gamma_{3}}{d u^{2}}+\frac{1}{4}\left(v^{2}+1-3 v^{2} s n^{2} u\right) \gamma_{3}=0$, whose general solution is $8 \mathrm{~K}$-periodic (see [29] Chapter 23, exercise 18).

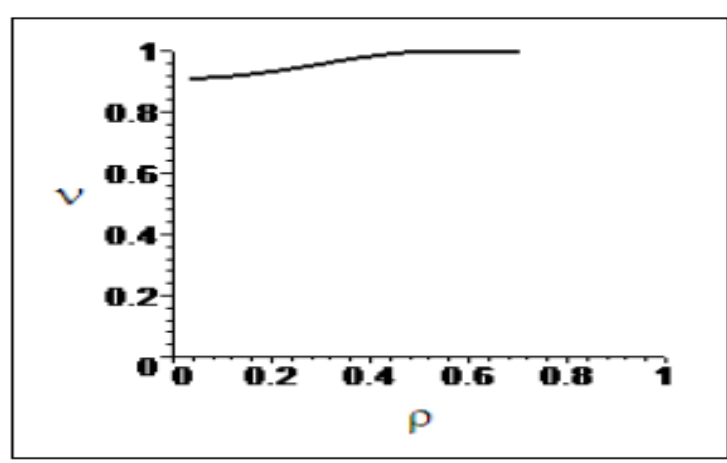

Fig. 4 The left face $\alpha=0+$

For $\alpha=4$ the zones of stability are shown in Fig. 6.

\section{Numerical study}

The linear periodic equation (20) may be written as:

$$
\chi^{\prime \prime}+\kappa \chi^{\prime}+\sigma \chi=0,
$$

where the dash refers to the derivative with respect to $\phi$ and $\kappa, \sigma$ have the form

$$
\begin{gathered}
\kappa=\frac{-v^{2} \sin \varphi \cos \varphi}{\left(1-v^{2} \sin ^{2} \varphi\right)}, \\
\sigma=\frac{\left(H+\frac{1}{2} \alpha(\alpha+1) v^{2} \cos 2 \varphi+2 v \rho \alpha^{\frac{3}{2}} \cos \varphi\right)}{\left(1-v^{2} \sin ^{2} \varphi\right)} .
\end{gathered}
$$

The study of stability of the three-term equation (30) is quite involved. The theory of periodic differential equations is much more developed for Hill's equation. The substitution

$$
\begin{aligned}
& \chi=\Phi y, \\
& \Phi=e^{-\frac{1}{2} \int \kappa d \varphi}=\left(1-v^{2} \sin ^{2} \varphi\right)^{\frac{-1}{4},}
\end{aligned}
$$

Transforms the periodic equation (30) to Hill's equation

$$
\begin{aligned}
& \frac{d^{2} y}{d \varphi^{2}}+Q(\varphi) y=0, \\
& Q=\left(\sigma-\frac{\kappa^{2}}{4}-\frac{\kappa^{\prime}}{2}\right) .
\end{aligned}
$$


Let $y_{1}, y_{2}$ be a fundamental set of solution of (34) satisfying the conditions

$$
\begin{array}{ll}
y_{1}(0)=1 & y_{2}(0)=0, \\
y_{1}^{\prime}(0)=0 & y_{2}^{\prime}(0)=1 .
\end{array}
$$

The zones of stability of the zero solution of (34) are characterized by the condition [30]

$$
\left|y_{1}(2 \pi)+y_{2}^{\prime}(2 \pi)\right|<2 \text {. }
$$

They are separated from instability zones by the boundaries

$$
\left|y_{1}(2 \pi)+y_{2}^{\prime}(2 \pi)\right|=2 \text {. }
$$

on which at least one solution is primitive periodic. Generally speaking, the second solution of (34) on (36) is not periodic due to the presence of a secular term, leading to instability of the zero solutions of (34). However, the second solution is also periodic on the intersection of two boundaries. In this case the zero solution is stable. Now, we transform the stability condition (36) to a condition on the solution of equation (30). Denote by $\chi_{1}, \chi_{2}$ the solution of (30) corresponding to the fundamental solutions of (34), i.e.

$$
\chi_{1}=\Phi y_{1} \quad, \quad \chi_{2}=\Phi y_{2} .
$$

We get the initial conditions

$$
\begin{array}{ll}
\chi_{1}(0)=\Phi(0) & , \quad \chi_{1}^{\prime}(0)=0, \\
\chi_{2}(0)=0 \quad, \quad \chi_{2}^{\prime}(0)=\Phi(0) .
\end{array}
$$

The stability condition (36) takes the final form

$$
\left|\left(\chi_{1}(2 \pi)+\chi_{2}^{\prime}(2 \pi)\right)\right|<2 .
$$

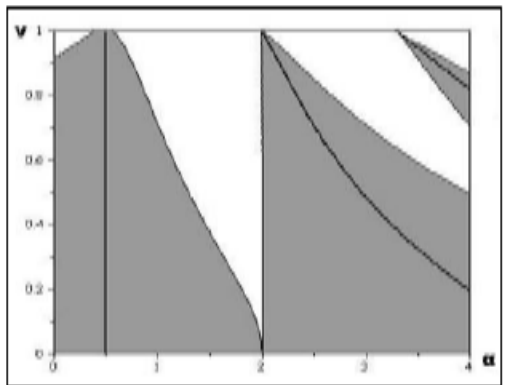

a: the face $\rho=0$

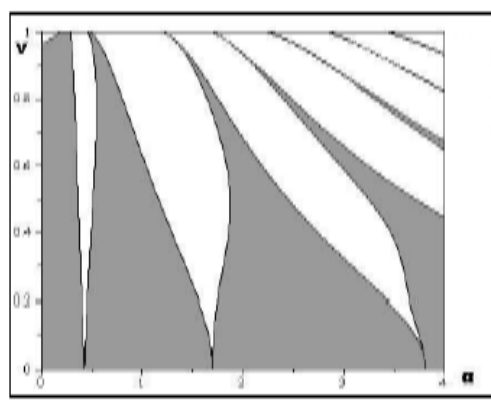

$\mathrm{d}: \rho=0.3$

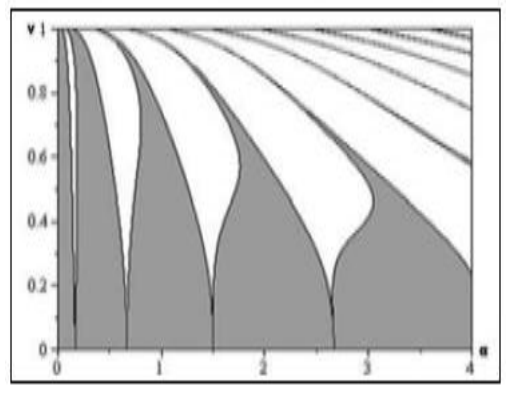

$\mathrm{g}: \rho=1$

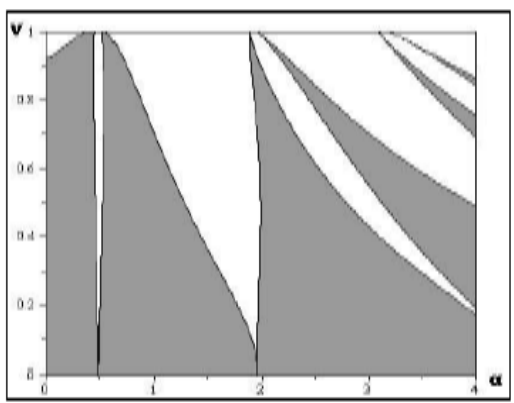

b: $\rho=0.1$

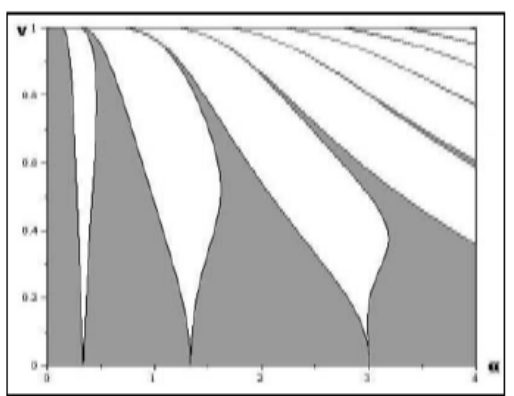

e: $\rho=0.5$

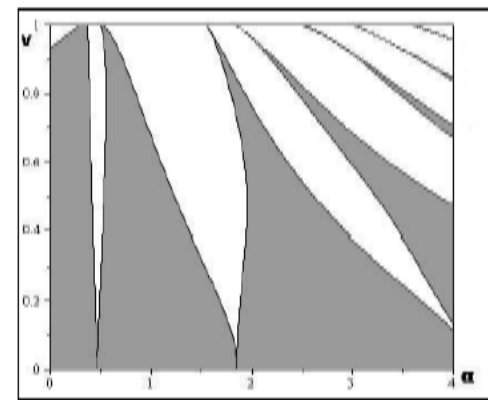

c: $\rho=0.2$

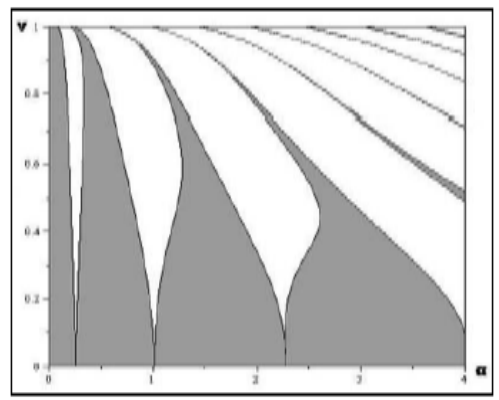

f: $\rho=0.7$

Stability zone

Instability zone

Fig. 5 Sections of stability zones at different levels of the gyrostatic parameter $\rho$

Fig. 7 represents the numerical results of stability analysis for special values of $\rho$ $(\rho=0,1)$. Black points represent mean stability of the corresponding vibration according to 
numerical calculation; while points corresponding to instability are left blank completely agree with the analytically calculated boundaries drawn in solid lines.

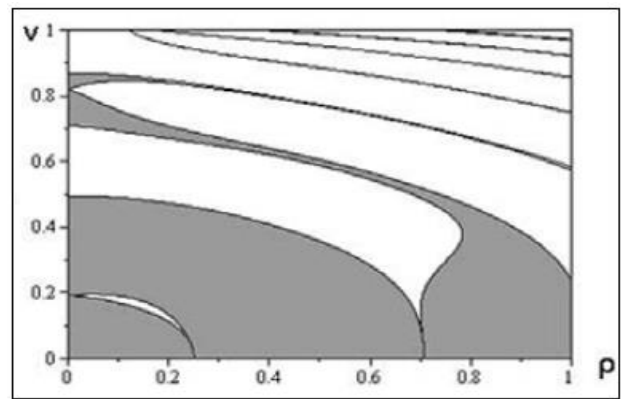

Fig. 6 The right face $\alpha=4$
Fig. 8 shows the numerical results of stability analysis for special values of $v \quad(v=0.3,0.5$, $0.7,0.9,0.95,0.97,0.99)$. Points represent points of stability according to numerical calculations. Points corresponding to instability are left blank. This completely agrees with the analytical result drawn in solid lines. Fig. (8-f) shows the numerical results of stability analysis for $v=0.99$ calculated from the determinants with 35 rows and columns, Fig. (8-h) for $v=0.99$ calculated from the determinants with 40 rows and columns. We note that for $v=0.99$ Fig. (8g) gives a good analytical approximation, in completely agreement with the numerical one.
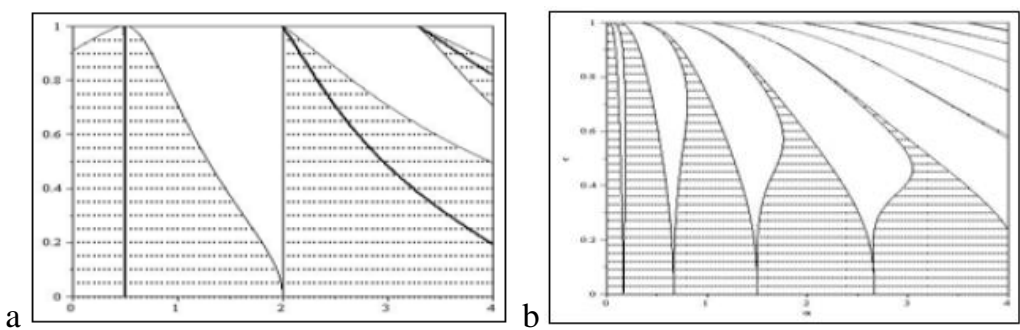

Fig. 7 Sections of stability zones at different levels of the gyrostatic parameter $\rho$ analytical and numerical. a: the front face $\rho=0 \quad$ b: $\rho=1$

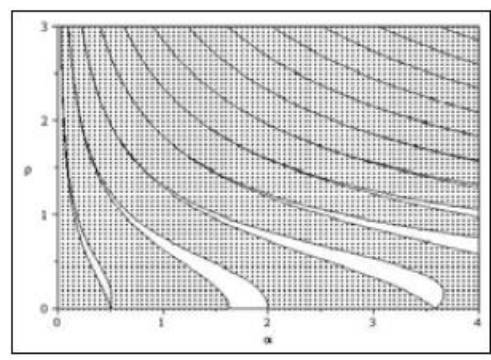

a: $v=0.3$

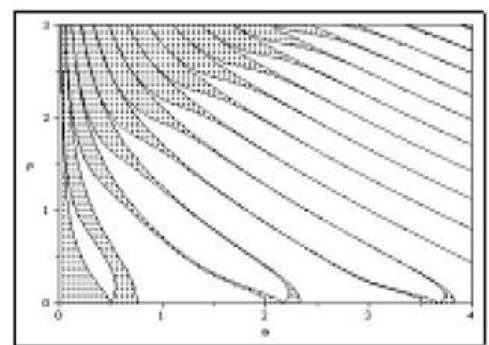

$\mathrm{d}: v=0.9$

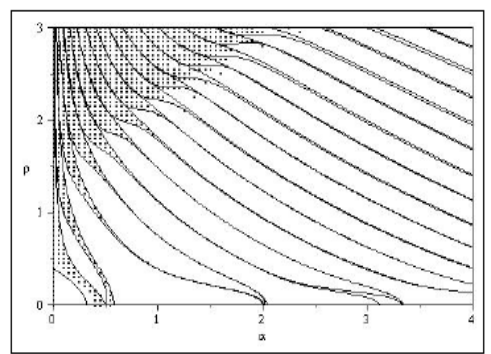

$\mathrm{g}: v=0.99$

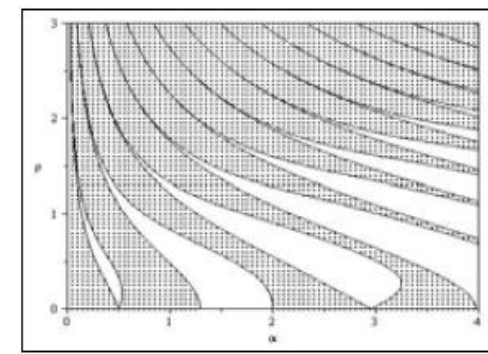

b: $v=0.5$

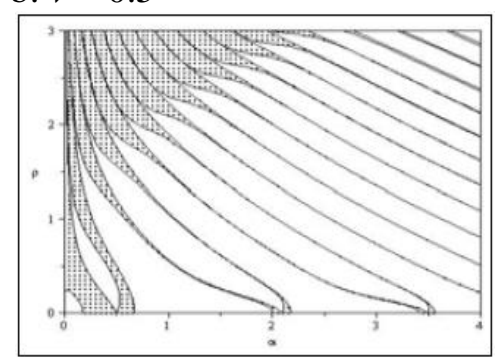

$\mathrm{e}: v=0.95$

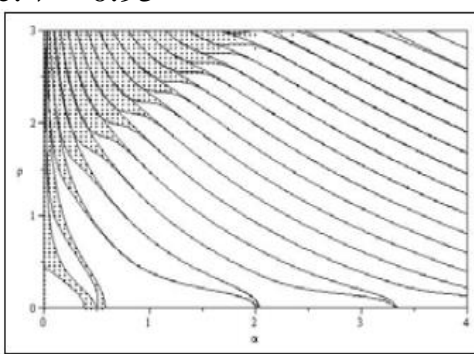

h: $v=0.99$

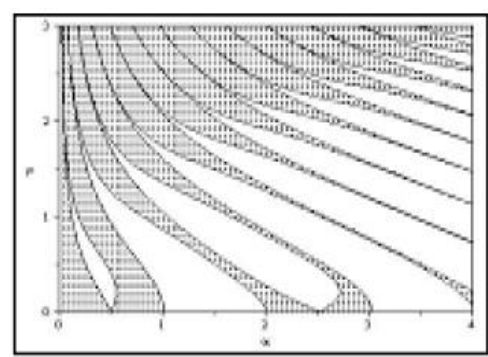

c: $v=0.7$

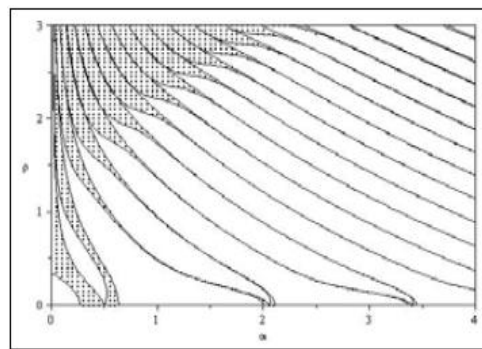

$\mathrm{f}: v=0.97$

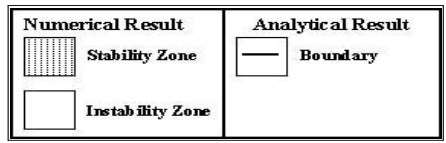

Fig. 8 Sections of stability zones at different levels of the modulus $v$ of the elliptic function 
The overall conclusion may draw of Fig. 8 is that with the increase of the modulus $v \in[0,1)$ instability zones spread from right and from below. By $v$ reaching 0.99 instability prevails for all bodies with oblate inertia spheroid, while several (different) stability zones still exist for the body with prolate inertia spheroid in the upper left corner of Fig. (8-h).

\section{Conclusion}

It is well known that the addition of the rotor along the axis of symmetry of a rigid body can stabilize if given suitable angular velocity the unstable (upper) position of equilibrium. Rotors are used as stabilizing factor in several situations in space dynamics. It is of great interest to study the effect of the added rotor on the stability of some simple periodic motions.

The orbital stability of the plane (pendulum-like) vibrations of a rigid body about a fixed point is analyzed. The variation equation is a form of Hill's equation

$$
\frac{d^{2} \gamma_{3}}{d u^{2}}+\alpha\left(\alpha v^{2}+\frac{1}{2}+\rho^{2}-(\alpha+1) v^{2} s n^{2} u+2 v \rho \sqrt{\alpha} \text { cnu }\right) \gamma_{3}=0,
$$

which generalizes Lame's equation by the presence of one additional parameter $\rho$ depending on the gyrostat moment. This equation is not treated in the literature to any extent.

Applying Floquet's theory to solve the periodic eigen-value problem for this equation we determine the boundaries of stability and instability zones in the admissible region of the 3D space $\{\alpha, v, \rho: 0<\alpha \leq 4,0 \leq v<1, \rho \geq 0\}$.

Analytically calculated boundary surfaces are plotted in $3 \mathrm{D}$ space and various plane sections are compared with numerically computed stability zones. Analytical and numerical results show good agreement.

It is noted that with the increase of the gyrostatic moment stability and instability zones are pressed to left and new zones appear from the right side $\alpha=4$. As $v$ increases, instability prevails for bodies with oblate inertia spheroid.

\section{References}

[1] S. Kovalevskaya, Sur le problème de la rotation d'un corps solide autour d'un point fixe. Acta
Math. 12 (1889) 177-232

[2] E. Leimanis, The General Problem of Motion of Coupled Rigid Bodies, Springer-verlag (1964)

[3] R. Grammel, Gyroscope. Theory and Applications Part I, II (1952) (In Russian)

[4] V.V. Rumyantsev, Stability of permanent rotations of a heavy rigid body, Prikl. Math. Mech. 20 (1956) (in Russian)

[5] W.O. Schiehlen, H.I. Weber, On the stability of Staude's permanent rotations with damping. Ingenieur-Archiv. 46 (1977)

[6] A.D. Bilimoich, Equations of motion of a heavy rigid body about a fixed point. Collection of papers devoted to Prof. G. K. Suslov, Kiev (1911) 23-74

[7] W. Hess, Über die Eulerschen Bewegungsgleichungen und eine neue particuläre lösung des problems der Bewegung eines starren schweren körpers um einen festen Punkt., Math. Ann. 37 (1890) 153-181

[8] P.A. Schiff, On equations of motion of a rigid body, Mat. Sbornik 24 (1903) 169-177

[9] P. Stäckel, Die reduzierten Differenzialeichungen der Bewegung des schweren unsymmetrichen Kreisels. Math. Ann. 67 (1909) 399-432

[10] E.I. Kharlamova, Reduction of the problem of motion of a body with a fixed point, to a single differential equation, Mekh. Tverdogo Tela 1 (1969) 107-116

[11] H.M. Yehia, On the reduction of the order of differential equations of motion of a rigid body about a fixed point, Vestnik MGU, Ser. Math. Mekh 6 (1976) (in Russian)

[12] H.M. Yehia, On the reduction of the equations of motion of a rigid body in an axisymmetric field, J. Mećan. Théor. Appl. 2 (1983) 451-462

[13] H.M. Yehia, Qualitative investigations of a rigid body about a fixed point, Prikl. Mat. Mech. 45 (1981) 454-458

[14] H.M. Yehia, On the stability of plane motions of a heavy rigid body about a fixed point. ZAMM. Z. Angew. Math. Mech. 67 (1987) 641-648

[15] H.M. Yehia, On the stability of plane motions of a rigid body about a fixed point in a Newtonian field of force, Vestn. MGU, Ser. Math. Mekh 4 (1981)

[16] V. Tkhai, A.L. Shvygin, On the stability of rotations about a horizontal axis of the heavy rigid body with a fixed point. In "Problems of investigation of stability and stabilization of motion"(Ed.V.Ramyantsev) Moscow, Computing centre of the Russian Academy of Science, Part 2, (2000) 149-157

[17] S.A. Dovbysh, Oscillational properties of plane motions in the dynamics of a symmetric rigid body. Izvest .R.A.N. Mekh. Tverdogo. Tela, No4 (1990) 11-19

[18] A.P. Markeev, Plane and quasi-plane rotations of a heavy rigid body about a fixed point, Izv. AN 
SSSR. Mekhanika Tverdogo Tela 23 (1988) 2936

[19] A.P. Markeev, The stability of the plane motions of a rigid body in the Kovalevskaya case, Prikl. Mat. Mehk 65 (2000) 51-58

[20] A.P. Markeev, The pendulum- like motions of a rigid body in the Goryachev-Chaplygin case, Prikl. Mat. Mehk 68 (2004) 282-293

[21] G. Grioli, Esistenza e determinazione delle precessioni regolari dinamicamente possibili per un solido pesante asimmetrico. Ann. Mat. Pure Appl. 4 (1947) 271-281

[22] A.Z. Bryum, Investigation of the regular precession of a heavy rigid body with a fixed point by Lyapunov's first method. Mechanics of Solids, Naukova Dumka, Kiev 19 (1987) 68-72

[23] G.V. Mozalevskaya, A.P. Kharlamov, E.I Kharlamova, Drift of G. Grioli's gyroscope. In Mechanics of Solids, Naukova Dumka, Kiev 24 (1992) 15-25
[24] V.N. Tkhai, The Stability of regular Grioli precessions. J. Appl. Math. Mehk. 64 (2000) 811-819

[25] A.P. Markeev, On stability of regular precessions of a non-symmetric gyroscope. Reg. Chaot. Dyn. 8 (2003) 297-304

[26] A.P. Markeev, The stability of the Grioli precession. J. Appl. Math. Meck. 67 (2003) 497510

[27] A.P. Markeev, On the Steklov case in rigid body dynamics, Reg. Chaot. Dyn. 10 (2005) 81-93

[28] A. Erdélyi, Higher Transcendental Functions, Mc Graw Hill (1953)

[29] E.T. Whittaker, Watson G.N., A course of modern analysis, 4th ed. (Reprinted), Cambridge University Press (1963)

[30] F. M. Arscott, Periodic differential equations, Pergamon Press (1964)

[31] E. L. Ince, The periodic Lame functions, Proc. Royal Soc. Edinburgh 60 (1940)

\section{استقرار الحركة التذبذبية لبندول ثقيل حول نقطة ثابتة}

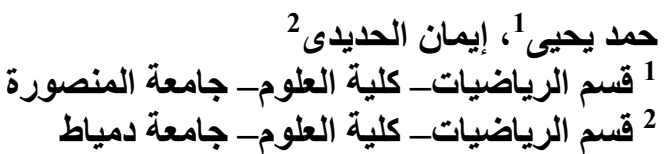

يهتم البحث بدراسة استقرار الحركات البندولية لجيروستات ثقيل حول نقطة ثابتة في حالة الحركة

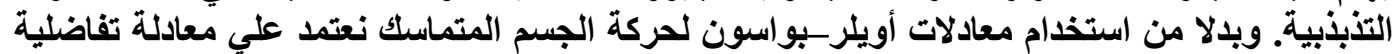

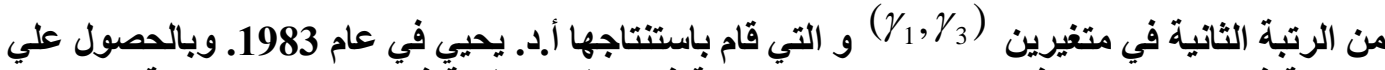

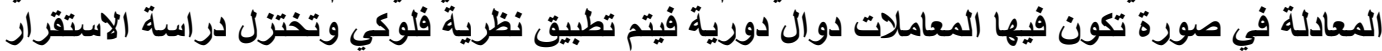

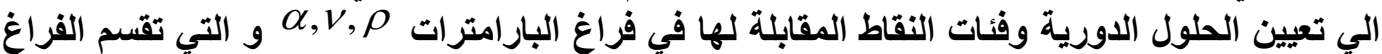

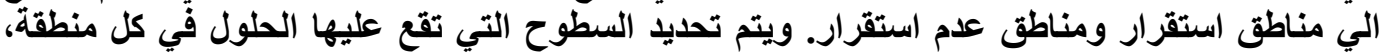

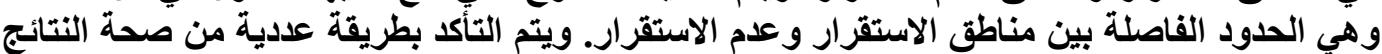
وترسم صورة كاملة للمناطق في فراغ لاغ البارامترات. 